\title{
Orientational transition in a nematic liquid crystal at a patterned surface
}

\author{
T. J. Atherton* and J. R. Sambles \\ Thin Fim Photonics Group, School of Physics, University of Exeter, Stocker Road, Exeter EX4 4QL, England
}

(Received 14 March 2006; revised manuscript received 7 June 2006; published 31 August 2006)

\begin{abstract}
We consider a semi-infinite nematic in contact with a periodic patterned surface with alternate planar and homeotropic stripes. Extending the work of Barbero et al., we find the free energy (assuming $K_{1}=K_{3}$ ) for the situations where the easy direction on the planar stripe is either perpendicular or parallel to the length of the stripes. We find the bulk free energy difference between the structures to be proportional to $\sqrt{K_{2} / K_{1}}$ and so we consider the possibility of a spontaneous transition between the two states if the azimuthal anchoring energy is sufficiently weak and $K_{1} \neq K_{2}$. We compute the critical azimuthal anchoring energy for such a transition in terms of the relative width of the stripes and the period of the pattern and find it to be $\sim 10^{-6} \mathrm{~J} \mathrm{~m}^{-2}$, comparable to experimental values.
\end{abstract}

DOI: 10.1103/PhysRevE.74.022701

PACS number(s): 61.30.Hn, 61.30.Gd, 61.30.Cz

\section{INTRODUCTION}

In recent years there has been much interest in the alignment of nematic liquid crystals with micropatterned and nanopatterned surfaces to construct bistable devices [1-3]. Using, for example, a photoaligned polymer [4] developed with appropriate masks, a surface may be fabricated which incorporates regions that promote either homeotropic or planar alignment. Near such a surface the director field $\hat{\mathbf{n}}$ is highly distorted; away from the surface the nematic adopts a uniform bulk orientation that depends on the relative area of the planar and homeotropic regions. It is therefore possible to pattern a surface that promotes any desired orientation in the bulk nematic.

One pattern that has been fabricated is a periodic array of stripes that alternately promote planar and homeotropic alignment. We restrict our attention in this paper to the cases where the preferred azimuthal aligment on the planar stripes is either parallel or perpendicular to the stripes. The case where the director lies perpendicular to the stripes we shall refer to as a "splay-bend" surface [Fig. 1(a)] and the case where the director is parallel to the stripes we refer to as a "twist" surface [Fig. 1(b)].

The splay-bend geometry has been studied theoretically: Barbero considered the uniform bulk orientation promoted by a splay-bend surface with arbitrary pretilt angles and finite polar anchoring [5]. Kondrat showed that for finite polar anchoring if either the planar or homeotropic set of stripes is very narrow, the nematic may adopt a spatially uniform configuration rather than the distorted configuration [6].

In this paper we extend previous work on the splay-bend surface to the twist surface. We show that if the twist elastic constant is lower than the splay and bend elastic constants, as is the case for most nematics, the energy of a nematic is lower if it is in contact with a twist surface than with a splay-bend surface. We consider the feasibility of a transition between the two states which might take place if the azimuthal anchoring on the planar stripes is rather weak, and

*Electronic address: t.j.atherton@ex.ac.uk compute the critical azimuthal anchoring energy as a function of period and relative homeotropic stripe width.

\section{MODEL}

The final configuration of the nematic is that which minimizes the total free energy including bulk and surface contributions. The bulk energy density is the well-known Frank free energy,

$$
f_{b}=\frac{1}{2}\left[K_{1}(\boldsymbol{\nabla} \cdot \hat{\mathbf{n}})^{2}+K_{2}(\hat{\mathbf{n}} \cdot \boldsymbol{\nabla} \times \hat{\mathbf{n}})^{2}+K_{3}|\hat{\mathbf{n}} \times \boldsymbol{\nabla} \times \hat{\mathbf{n}}|^{2}\right]
$$

where $K_{1}, K_{2}$, and $K_{3}$ are the elastic constants associated with splay, twist, and bend distortions, respectively, and are all of order $10^{-11} \mathrm{~N}$ except near phase transitions. The saddle-splay $K_{24}$ term is omitted as it does not contribute to the free energy if the director is confined to a single plane.

The nematic-surface interaction is characterized by an anchoring potential that quantifies the energy cost of moving the director away from an "easy" direction. A commonly used phenomenological potential is the Rapini-Papoular potential [7],

$$
f_{s}=\frac{1}{2}\left[W_{\theta} \sin ^{2}\left(\theta-\theta_{e}\right)+W_{\phi} \cos ^{2} \theta \sin ^{2}\left(\phi-\phi_{e}\right)\right],
$$

where $W_{\theta}$ is the polar anchoring energy, $W_{\phi}$ is the azimuthal anchoring energy, and the two angles $\theta_{e}$ and $\phi_{e}$ define the easy orientation. Experimentally determined values of $W_{\theta}$ are of order $1 \times 10^{-4} \mathrm{~J} \mathrm{~m}^{-2}$ [8]. Typically, $W_{\phi}$ is found to be an order of magnitude lower than $W_{\theta}$ [9-11].

To evaluate the free energy, we adopt a coordinate system in which the $x$ coordinate is along the surface and perpendicular to the length of the stripes and the $z$ coordinate is normal to the surface; both coordinates are scaled by the period of the pattern $\lambda$. If the director is constrained to a single plane that makes an azimuthal angle $\phi$ with the wave vector of the stripes, the director may be parametrized,

$$
\hat{\mathbf{n}}=\{\cos \theta \cos \phi, \cos \theta \sin \phi, \sin \theta\} .
$$


(a)
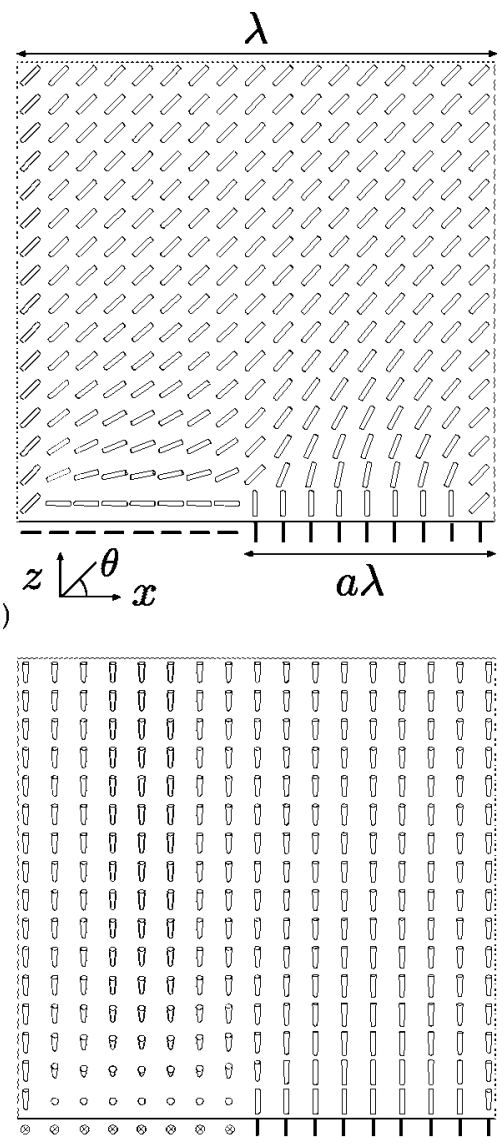

(b)

FIG. 1. Schematic of the director field $\hat{\mathbf{n}}(x, z)$ for a nematic with $K_{1}=K_{2}=K_{3}$ in contact with a surface patterned with period $\lambda$ and relative homeotropic stripe width $a$. (a) "Splay-Bend" surface; (b) "Twist" Surface.

For most nematics $K_{2}<K_{1} \leq K_{3}$ and we therefore make the simplification that $K_{1}=K_{3}$. The free energy density is then

$$
f / K_{1}=\frac{1}{2}\left(\kappa \theta_{x}^{2}+\theta_{z}^{2}\right)
$$

where $\kappa=\left[1-(1-\tau) \sin ^{2} \phi\right]$ and $\tau=K_{2} / K_{1}$. The EulerLagrange equation

$$
\kappa \theta_{x x}+\theta_{z z}=0
$$

has a Fourier series solution that is regular as $z \rightarrow \infty$,

$$
\begin{aligned}
\theta(x, z)= & \theta_{0}+2 \sum_{n=1}^{\infty} \exp (-2 n \pi \sqrt{\kappa z})\left[p_{n} \sin (2 n \pi x)\right. \\
& \left.+q_{n} \cos (2 n \pi x)\right] .
\end{aligned}
$$

The coefficients $p_{n}$ and $q_{n}$ are to be determined from the boundary conditions at the surface. Substituting Eq. (6) into (4) and integrating over $x$ and $z$ yields the bulk free energy per period,

$$
F / K_{1}=4 \pi \sqrt{\kappa} \sum_{n=1}^{\infty} n\left(p_{n}^{2}+q_{n}^{2}\right) .
$$

If the Fourier coefficients are nearly independent of $\tau$ as we show below and $K_{2}<K_{1}$, Eq. (7) has a minimum at $\phi$ $=\pi / 2$, which corresponds to the twist geometry, and a maximum at $\phi=0$ which corresponds to the splay-bend geometry. The energy of a nematic in contact_with each of the two geometries differs by a factor of $1-\sqrt{\tau}$. It is possible then for a nematic with $K_{2}<K_{1}$ in contact with a splay-bend surface to spontaneously "twist out" to adopt the twist structure if the azimuthal anchoring is sufficiently weak. Conversely, a nematic with $K_{2}>K_{1}$ in contact with a twist surface may spontaneously adopt the splay-bend configuration. The necessary criterion in both cases is that the energy difference between the two configurations in the bulk is greater than the energy cost at the surface,

$$
\left|F_{S B}(a, \lambda)-F_{T W}(a, \lambda, \tau)\right|>W_{\phi} \lambda(1-a) / K_{1} .
$$

Neither the twist nor splay-bend configuration (depending on the ratio $K_{2} / K_{1}$ ) necessarily represents the absolute energy minimum: it is conceivable that the energy may be reduced by a periodic modulation of $\phi$; spontaneous twist modulations occur in other liquid crystal systems $[12,13]$ but they require very anisotropic elastic constants $\left(K_{2} / K_{1}\right.$ $<0.3$ ) or submicron cell thickness. We restrict our attention to common nematic materials in which the amplitude of such a modulation in $\phi$ will necessarily be very small and so Eq. (7) is a good estimate of the free energy. If the elastic constants are very anisotropic, the possibility of a modulation in $\phi$ might be explored by a variational method [13] or numerical solution of the Euler-Lagrange equations.

\section{RESULTS}

We first consider the situation where $\theta$ is fixed along the surface,

$$
\theta(x, 0)=\left\{\begin{array}{ll}
\frac{\pi}{2} & 0 \leq x<a \\
0 & a \leq x<1
\end{array},\right.
$$

then $\theta_{0}$ is simply the average value $\langle\theta(x, 0)\rangle=\pi a / 2$ and the coefficients $p_{n}$ and $q_{n}$ are the coefficients in the Fourier series,

$$
p_{n}=\frac{\sin ^{2}(n a \pi)}{2 n}, \quad q_{n}=\frac{\sin (2 n a \pi)}{4 n} .
$$

The associated free energy

$$
F=\pi \sqrt{\tau} \sum_{n=1}^{\infty} \frac{\sin (n a \pi)^{2}}{n}
$$

does not converge due to the poles in $\nabla \theta$ at $(x, z)=(0,0)$ and $(a, 0)$ which, in this rigid anchoring model, are disclination lines.

To deal with similar cases, others have postulated that above a certain magnitude of $\nabla \theta$, the nematic "melts" leav- 


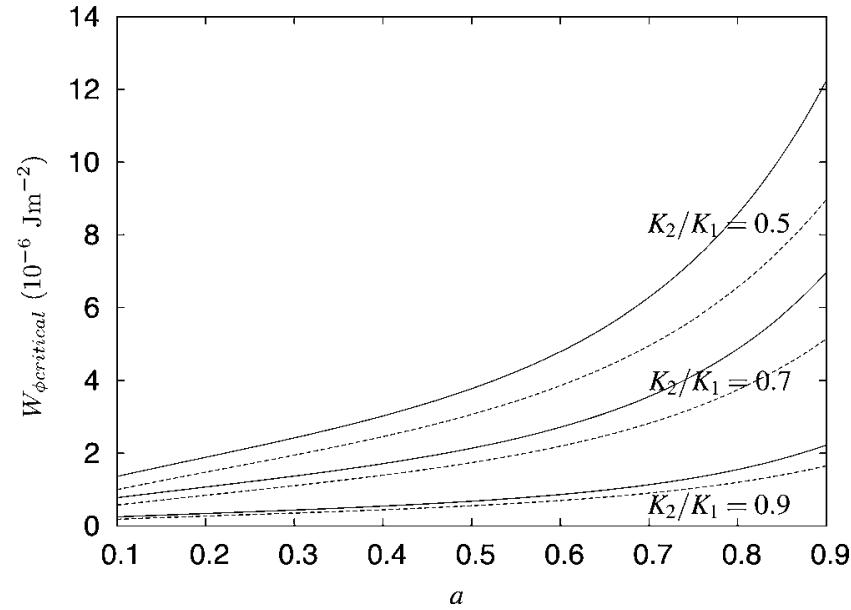

FIG. 2. Critical azimuthal anchoring energy as a function of $a$ for $\lambda=10 \mu \mathrm{m}, K_{1}=1 \times 10^{-11} \mathrm{~N}$, and with rigid polar anchoring. Solid lines were computed with $r_{c}=10^{-8} \mathrm{~m}$ and dashed lines with $r_{c}=5 \times 10^{-8} \mathrm{~m}$. Note that a transition from splay-bend to twist is feasible if $W_{\phi}<W_{\phi c r i t i c a l}$.

ing a small isotropic region at the core of the disclination. We assume that the core energy of the disclinations is independent of $\tau$ and define a cutoff radius $r_{c}$ (a typical value is $10^{-8} \mathrm{~m}$ [14]) below which the elastic theory breaks down. The free energy density (4) is locally convergent except at the poles and we may therefore integrate it numerically over $x$ and $z$ excluding the two semicircles around the disclinations defined by $x^{2}+z^{2}<r_{c}^{2}$ and $(x-a)^{2}+z^{2}<r_{c}^{2}$.

The azimuthal anchoring energy which just satisfies Eq. (8) for the splay-bend to twist transition is plotted in Fig. 2 as a function of $a$ for several values of $\tau$. An equivalent plot for the twist to splay-bend transition may be obtained by relabelling $K_{2} / K_{1} \rightarrow K_{1} / K_{2}$. A second plot of $W_{\phi c r i t i c a l}$ with respect to $\lambda$ shows that reducing the period also favours the transition (Fig. 3). If the period is short, $a$ may be restricted in fabrication as the minimum width of a stripe developed with a mask is $\approx 1 \mu \mathrm{m}$. Comparing our calculated $W_{\phi c r i t i c a l}$ with experimentally measured values, we see that it is simi-

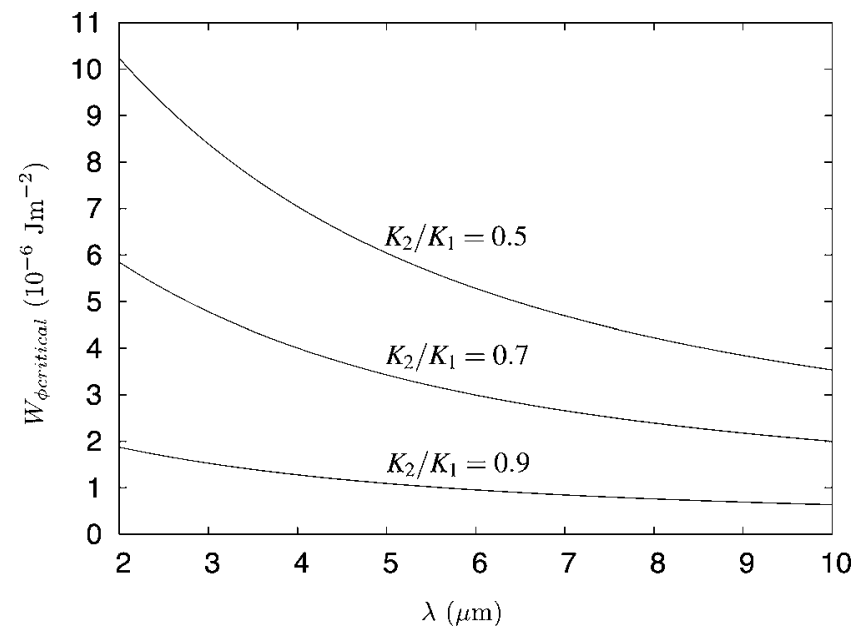

FIG. 3. Critical azimuthal anchoring energy as a function of $\lambda$ with rigid anchoring for $a=0.5, r_{c}=10^{-8} \mathrm{~m}$, and $K_{1}=1 \times 10^{-11} \mathrm{~N}$. lar to that obtained for obliquely evaporated silicon oxide $\left(W_{\phi}=2.9 \times 10^{-6} \mathrm{~J} \mathrm{~m}^{-2}[11]\right)$ and photoaligned polymer films $\left(W_{\phi}<1 \times 10^{-6} \mathrm{~J} \mathrm{~m}^{-2}[10]\right)$ and so we might expect to observe the transition with these surface treatments; somewhat larger values of $W_{\phi}\left(2.3 \times 10^{-5} \mathrm{~J} \mathrm{~m}^{-2}\right.$ [9]) are measured for rubbed polyimide films and so we would not expect to observe the transition on such a surface. In a physical system, no fine boundary will exist between the stripes due, for example, to weak polar anchoring (which we consider below), misregistration in fabrication, and order parameter reduction. The latter effects are tantamount to changing the defect radius $r_{c}$ and we plot also in Fig. 2 the calculation with $r_{c}=5$ $\times 10^{-8} \mathrm{~m}$; the effect on $W_{\phi c r i t i c a l}$ is not sufficient to alter our conclusions.

We now consider the situation where $\theta$ is allowed to vary at the surface. We make the simplifying approximation that the azimuthal part of the anchoring energy is independent of $\theta$. Furthermore, in the Rapini-Papoular potential $\theta-\theta_{e}$ appears in the argument of a transcendental function and so it is not possible to evaluate the coefficients independently: we use an approximate potential $W_{\theta}\left(\theta-\theta_{e}\right)^{2} / 2$ which gives the boundary condition

$$
\left[\frac{\partial \theta}{\partial z}-2 \frac{\lambda}{L_{\theta}}\left(\theta-\theta_{e}\right)\right]_{z=0}=0,
$$

where $L_{\theta}=K_{1} / W_{\theta}$ is the penetration depth of the surface treatment. Substituting Eq. (6) into Eq. (12) and integrating we obtain the Fourier coefficients

$$
p_{n}=\frac{\sin ^{2}(n a \pi)}{2 n\left(1+2 n L_{\theta} \pi \sqrt{\tau}\right)}, \quad q_{n}=\frac{\sin (2 n a \pi)}{4 n\left(1+2 n L_{\theta} \pi \sqrt{\tau}\right)}
$$

and the associated free energy

$$
F=\pi \sqrt{\tau} \sum_{n=1}^{\infty} \frac{\sin (n a \pi)^{2}}{n^{2}\left(1+2 L_{\theta} n \pi \sqrt{\tau}\right)}
$$

is convergent and may be used to evaluate the critical azimuthal anchoring energy directly. Quantitatively similar results for $W_{\phi c r i t i c a l}$ as a function of $a$ and $\lambda$ to those calculated from the rigid anchoring model are obtained for $W_{\theta}=1$ $\times 10^{-4} \mathrm{~J} \mathrm{~m}^{-2}[8]$. The polar anchoring strength in the weak polar anchoring model has the same effect as changing the defect core radius $r_{c}$ in the strong anchoring model as the energy difference between the splay-bend and twist states depends on how well the surface treatment is able to maintain the large director gradient around the boundary between adjacent stripes. $W_{\phi c r i t i c a l}$ grows logarithmically with $W_{\theta}$ (Fig. 4).

A restriction on $a$ occurs as $a \rightarrow 1$ in the finite polar anchoring model, a transition to a uniform homeotropic state as predicted by Kondrat [6] will occur for some critical $a$; the "splay-bend-twist" transition discussed in this paper will raise the critical $a$ for which the uniform state has lower energy.

\section{CONCLUSION}

We have considered the bulk energy difference of a semiinfinite nematic region in contact with each of the splay-bend 


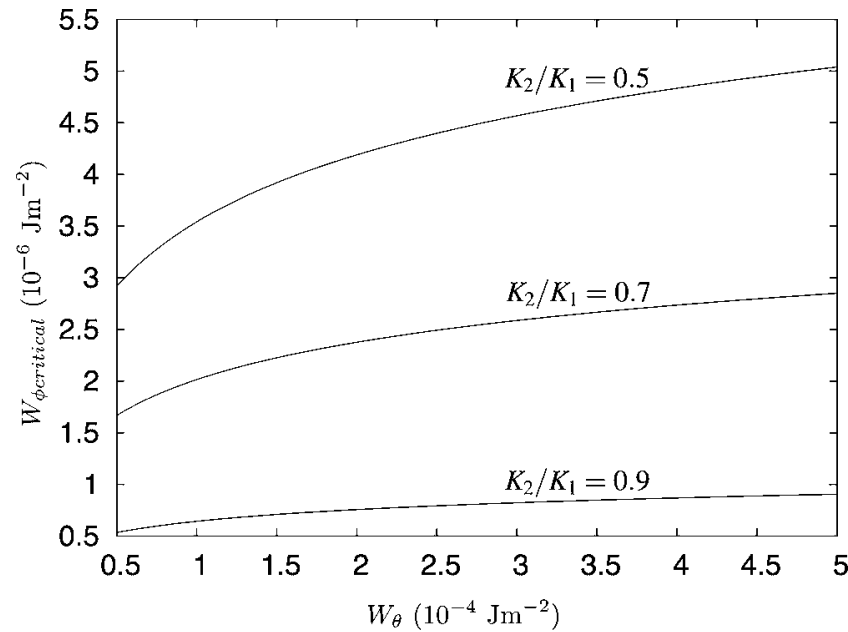

FIG. 4. Critical azimuthal anchoring energy as a function of the polar anchoring energy $W_{\theta}$ for $a=0.5, \lambda=10 \mu \mathrm{m}$, and $K_{1}=1$ $\times 10^{-11} \mathrm{~N}$.

and twist patterned surfaces for anisotropies in the elastic constants consistent with those measured in typical liquid crystal compounds (i.e., $1 / 2<K_{2} / K_{1}<2$ ). The energy difference is sufficient to make a transition feasible between the two structures if the Rapini-Papoular azimuthal anchoring energy is less than $10^{-5} \mathrm{~N} \mathrm{~m}^{-2}$ : the transition is energetically favored for surfaces designed to promote high pretilt angles where the planar stripe width is small compared to the homeotropic stripe widths (i.e., as $a \rightarrow 1$ ); the transition is also favored if the period $\lambda$ is made smaller.
Although we considered a semi-infinite nematic, we anticipate that the transition will occur in a physical system: the elastic distortion vanishes at a distance of the order of the penetration depth $L=\lambda \sqrt{K_{1} / K_{2}} /(2 \pi)$ and so if the nematic is terminated by a second interface at a distance $d \gg L$ the perturbation of the director field in the distorted region is negligible. If the second surface is micropatterned identically to the first, then the stability criterion (8) still applies where the splay-bend and twist states are replaced by symmetric splaybend and twist states near either surface separated by a region of undistorted nematic in between.

The possibility of observing such a transition in a cell depends on the validity of the phenomenological azimuthal anchoring potential for large displacements from the easy orientation and the Rapini-Papoular energy is an underestimate. Grooved surfaces (where $W_{\phi} \approx 10^{-5} \mathrm{~J} \mathrm{~m}^{-2}$ ) are unlikely to allow the transition unless the polar anchoring energy is also much higher than $10^{-4} \mathrm{~J} \mathrm{~m}^{-2}$; the transition is likely to be possible on a photoaligned polymer surface $W_{\phi}$ $<10^{-6} \mathrm{~J} \mathrm{~m}^{-2}$.

A further interesting consequence is that a micropatterned surface with azimuthally degenerate planar stripes will nonetheless tend to align a typical nematic with $K_{2}<K_{1}$ along the length of the stripes as the twist state has lower energy. This mechanism requires anisotropic elastic constants unlike the well-known one due to Berreman [15].

\section{ACKNOWLEDGMENTS}

The authors would like to acknowledge the EPSRC and Sharp Laboratories of Europe for financial support and N. J. Smith of Sharp for helpful discussion.
[1] J. Kim, J. Yoneya, J. Yamamoto, and H. Yokoyama, Appl. Phys. Lett. 78, 3055 (2001).

[2] J. Kim, M. Yoneya, and H. Yokoyama, Nature (London) 420, 159 (2002)

[3] B. Zhang, F. K. Lee, O. K. C. Tsui, and P. Sheng, Phys. Rev. Lett. 91, 215501 (2003).

[4] M. Schadt, K. Schmitt, V. Kozinkov, and V. Chigrinov, Jpn. J. Appl. Phys., Part 1 21, 2155 (1992).

[5] G. Barbero, T. Beica, A. L. Alexe-Ionescu, and R. Moldovan, J. Phys. II 2, 2011 (1992).

[6] S. Kondrat, A. Poniewierski, and L. Harnau, Eur. Phys. J. E 10, 163 (2003).

[7] A. Rapini and M. Papoular, J. Phys. (Paris), Colloq. 30, C4-54 (1969).
[8] F. Yang, J. R. Sambles, Y. Dong, and H. Gao, J. Appl. Phys. 87, 2726 (2000)

[9] F. Yang, J. R. Sambles, and G. W. Bradberry, J. Appl. Phys. 85, 728 (1999).

[10] X. T. Li, D. H. Pei, S. Kobayashi, and Y. Iimura, Jpn. J. Appl. Phys., Part 2 36, L432 (1997).

[11] B. T. Hallam, F. Yang, and J. R. Sambles, Liq. Cryst. 26, 657 (1999).

[12] F. Lonberg and R. B. Meyer, Phys. Rev. Lett. 55, 718 (1985).

[13] O. Lavrentovich and V. M. Pergamenschik, Mol. Cryst. Liq. Cryst. 179, 125 (1990).

[14] S. Chandrasekhar, Liquid Crystals, 2nd ed. (Cambridge University Press, Cambridge, England, 1992).

[15] D. W. Berreman, Phys. Rev. Lett. 28, 1683 (1972). 